\title{
Design and production of the digital optical module of the KM3NeT project
}

\author{
Emanuele Leonora $^{1 \mathrm{a}}$, Valentina Giordano $^{1}$ \\ on behalf of the KM3NeT collaboration \\ ${ }^{1}$ INFN, sezione di Catania, Via S. Sofia, 64, 95123, Catania, Italy
}

\begin{abstract}
The KM3NeT collaboration is building the ARCA and ORCA neutrino telescopes in the depths of the Mediterranean Sea. They will consist of 3-dimensional arrays of photodetectors, called digital optical modules, suspended in the sea by means of vertical string structures, called detection units. The optical modules are composed of a pressure-resistant 17inch spherical glass vessel, which contains 31 small photomultiplier tubes and all the associated electronics. The multi- photomultiplier solution represents an innovative design with respect to optical modules of all currently operated neutrino telescopes comprising a single large photomultipliers.
\end{abstract}

\section{Introduction}

The KM3NeT Collaboration [1] started building a research infrastructure in the depths of the Mediterranean Sea hosting new generation neutrino telescopes located at the bottom of the Mediterranean Sea. The detectors of the KM3NeT telescopes can be described as a three dimensional matrix of photosensors that are sensitive to the Cherenkov radiation emitted by products of neutrino interactions in the deep water. The measured arrival time of the Cherenkov light at each sensor and the knowledge of their spatial position are used to reconstruct the trajectory of the electrically charged particle that produces the Cherenkov light. The amount of detected light provides information about the energy of the particle. The photosensors are called Digital Optical Modules (DOMs). In the sea, they are suspended in vertical structures, called Detection Units (DUs), supported by two Dyneema ${ }^{\circledR}$ ropes, anchored to the seabed and kept taut with a system of buoys. Following its first phase of construction, KM3NeT will comprise two detectors with different density of the arrays of optical modules: KM3NeT/ARCA (Astroparticle Research with Cosmics in the Abyss) at the KM3NeT-It site, located at the South of the Sicilian coast, will consist of two detector building blocks of 115 detection units each and will be dedicated to the detection of high-energy neutrino astronomy. KM3NeT/ORCA (Oscillation Research with Cosmics in the Abyss), a single more densily instrumented building block located at the KM3NeT-Fr site, offshore Toulon, will be dedicated to a lower neutrino energy range for study of neutrino mass hierarchy. The DOMs are pressure-resistant 17-inch diameter transparent spherical glass vessels, each hosting 31 photomultiplier tubes (PMTs) with a 3-inch photocathode diameter and all associated front-end and readout electronics. The multiPMT solution represents an innovative design with several advantages over traditional optical

\footnotetext{
${ }^{\text {a } C o r r e s p o n d i n g ~ a u t h o r: ~ e m a n u e l e . l e o n o r a @ c t . i n f n . i t ~}$
} 
modules with single large PMTs. The DOM design and its physics potential have been proven in-situ by several prototypes and in several detection units [2][3]. The DOM design phase is thus concluded successfully and mass production of KM3NeT DOMs is currently on going.

\section{The digital optical module design}

The Multi-PMT design of the KM3NeT optical module [4] has several advantages over traditional optical modules of all the other currently operating neutrino telescopes- ANTARES, Baikal and IceCube - which have optical modules with a single large photomultiplier, typically with a photocathode diameter of $10 \mathrm{inch}$. The total photocathode area comprised in the module is almost three times of that of a 10" PMT. The influence of the Earth's magnetic field on small size PMT is negligible and thus magnetic shield is not required. The Multi-PMT design, in which each sensor works individually, allows also for an accurate photon counting, because the arrival of more than one photon at the DOM can be identified with high efficiency. In addition, the segmented layout provides an almost isotropic field of view and efficient rejection of optical background from the bioluminescence and ${ }^{40} \mathrm{~K}$ in the sea just at the level of the DOM detection. In Fig. 1 a picture of a complete DOM is shown.

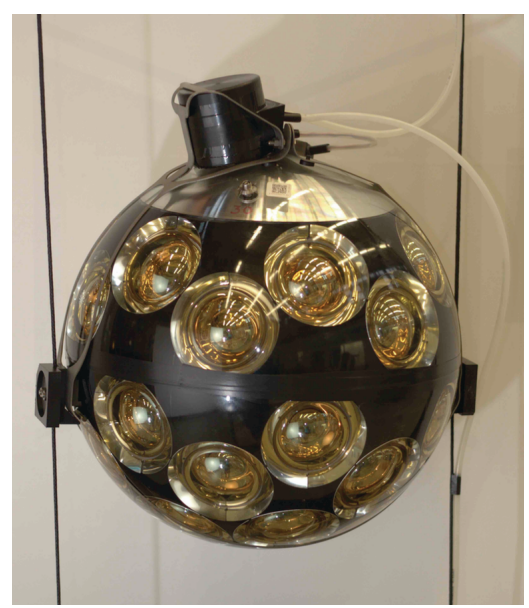

Figure 1. Picture of a DOM. The external collar, the break out box of the VEOC (Vertical Electrical-Optical Cable) backbone cable and the two parallel ropes of the detection unit are visible.

\subsection{The main components of the DOM}

The Digital Optical Module is composed of a transparent 17-inch diameter pressure resistant glass sphere housing 31 photomultiplier tubes and all the electronics. The transparent vessel is a Vitrovex ${ }^{\circledR}$ glass sphere, produced by Nautilus, composed of two separate hemispheres. The 31 PMTs are arranged in 5 rings of 6 PMTs plus a single PMT at the bottom pointing vertically downwards. The lower hemisphere contains 19 PMTs, the upper hemisphere 12 PMTs. The PMTs are kept in place by a 3D-printed support structure. The photon collection efficiency is increased by $20-40 \%$ by a reflector ring mounted around the face of each PMT. It allows increasing the detection area avoiding the use of a larger photocathode PMT.

The space between the support structure, the PMT windows and the glass sphere is filled with an optical silicone gel, in order to ensure an efficient mechanical and optical coupling between the different elements. It is a Silgel $612 \mathrm{~A} / \mathrm{B}$ produced by Wacker. The support structure and the gel have to be sufficiently flexible to be resistant to vibration and shocks during handling and deployment and allow for the deformation of the glass sphere under the hydrostatic pressure in the deep sea. 
In the Multi-PMT design, each PMT works as an individual optical sensor, with an individual lowpower high-voltage base with integrated amplification and tunable discrimination. The power dissipated of each base is below of $5 \mathrm{~mW}$. The photon arrival time and the time-over-threshold (ToT) of each PMT are recorded by an individual time-to-digital converter implemented in a Field Programmable Gate Array (FPGA). The threshold is set at the level of $1 / 3$ of the mean single photon pulse height and the high voltage is set to provide a gain of $3 \times 10^{6}$. The FPGA is mounted on the central logic board (CLB), which transfers the data to shore via an Ethernet network of optical fibers. The data provided by the PMT bases is collected and distributed to the CLB by means of two socalled Octopus Boards. The power board provides the DC power for all the systems inside the DOM. This board is monitored and controlled by the FPGA. It is powered with $12 \mathrm{~V}$ DC supplied via a penetrator mounted in the upper hemisphere of the DOM and connected to a DC/DC converter in the break-out-box external to the glass sphere (see Fig.1). An inner view of the DOM is shown in Fig. 2.

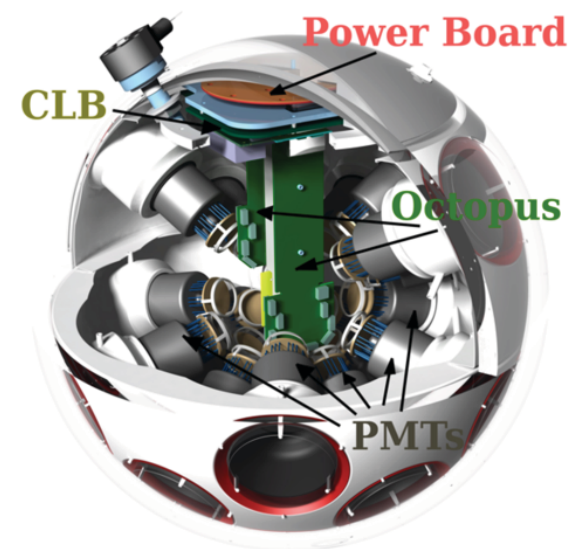

Figure 2. Sketch of the inner components of the DOM. CLB, power board, octopus boards and PMTs are indicated.

Each DOM in a detection unit communicates at a dedicated wavelength, which is multiplexed with other DOM wavelengths for transfer via a single optical fiber to the shore. The broadcast of the onshore clock signal, needed for time stamping in each DOM, is embedded in the Gb-Ethernet protocol. The White Rabbit protocol [5] has been modified to implement the broadcast of the clock signal, and allows for synchronization of all the DOMs of the KM3NeT detector with $1 \mathrm{~ns}$ resolution. An Aluminium structure provides heat conduction between the electronics inside and the environment outside of the sphere. Temperature and humidity sensors are mounted on the CLB and on the power board. The power consumption of a single DOM is about $7 \mathrm{~W}$.

For the first phase of the project, a batch of 15000 3-inch Hamamatsu PMT R12199-02 was calibrated. The PMTs have a convex bialkali photo-cathode, with a diameter of $80 \mathrm{~mm}$ and a 10 -stage dynode structure [6].

The DOM also contains three calibration sensors: a LED nano-beacon, which injects light in water to illuminate the neighbouring optical modules for time calibration; an acoustic piezo sensor is glued to the inner surface of the glass sphere to define the DOM position in water by means of an acoustic positioning system; an Attitude and Heading Reference System (AHRS) mezzanine board, mounted on the CLB, provides compass, tilt- and accelerometer data, to reconstruct the orientation of the DOM in the water.

A penetrator mounted in the upper hemisphere of the DOM contains two power cables and one optical fibre for data transmission. An external titanium collar holds the DOM to the two parallel Dyneema ropes of the string into the water. More details of the DOM design can be found in the Letter of Intent for KM3NeT 2.0 [7] 


\subsection{DOM main production phases}

Each DOM is composed of over 80 different elements: mechanical objects, electronics and sensors. Therefore, the production of the DOM is a delicate process performed following a well-defined procedure. Many tools are used, and a lot of them have been custom designed by the KM3NeT collaboration.

Each DOM integration site in the Collaboration receives all the components, which meet acceptance criteria and have already passed characterization tests. During the assembly phases, all the components of each DOM are registered in database by means of a dedicated assembly software. As the glass vessel is composed of two halves, the two parts of the DOM, top and bottom, are constructed separately. A lot of the DOM integration steps can be performed in parallel to speed up the production. Also the PMT support structures are segmented in two halves, each one made of two parts. The white 3-D printing structures have to be black painted before being glued together. All the components are carefully cleaned before their integration following the properly method defined in the procedure. In the upper hemisphere an aluminium mushroom-shaped structure is glued to the inner glass sphere by means of a special tool that define its height and the distance with the inner surface of the glass. Power board and CLB are later mounted on the inner part of the aluminium mushroom. The penetrator is mounted in the top glass sphere, and its water tightness is tested by means of a helium leakage detector. The optical fiber coming from the penetrator is connected to the laser transceiver operating an optical splice on the fiber. The resulting optical power and attenuation are checked, and results are stored in the database. The piezo sensor is glued to the inner surface of the bottom glass hemisphere. All the PMTs are mounted in the PMT structures, and their positions are stored in the database. After the structures equipped with all the PMTs are placed into the glass hemispheres, all the bases are connected to the octopus boards, and a functional test of all systems is performed before applying the optical gel, which joins optically and mechanically all the components together.

At the end of the integration process, the DOM is closed connecting the two halves setting down one part upon the other by means of an electrical crane. After closure, 0.2 bar of under-pressure inside the DOM, followed by the application of a sealant and a special tape on the junction of the two halves, closes the DOM. The external titanium collars is later mounted on the already closed DOM.

Each produced optical module is submitted to a final acceptance test and calibration of all the sensors performed in a light-tight dark box. Test and calibration data are later stored in the database of the KM3NeT Collaboration.

\section{Conclusions}

An innovative Multi-PMT digital optical module was designed as the sensitive part of the cubickilometer sized KM3NeT neutrino telescope. The design has been demonstrated in-situ by several prototypes and the mass production of the KM3NeT DOMs is on-going in several integration sites. Installation of detection units in the deep Mediterranean Sea has already started; two fully equipped detection units are taking data at the KM3NeT-It site offshore Porto Palo di Capo Passero, Sicily.

\section{References}

1. KM3NeT TDR, ISBN 978-90-6488-033-9, http://km3net.org.

2. S. Adrián-Martínez et al., Eur. Phys. J. C, 74, 3056 (2014), arXiv:1405.0839 [astro-ph.IM]

3. S. Adrián-Martínez et al., Eur. Phys. J. C, 76:54 (2016), DOI: 10.1140/epjc/s10052-015-3868-9

4. R. Bruijn and D. van Eijk for the KM3NeT Coll., in Proc. 34th Int. Cosmic Ray Conference, PoS (ICRC2015) 1157

5. Open Hardware Repository/CERN. http://www.ohwr.org/projects/white-rabbit

6. S. Aiello et al., AIP Conf. Proc. 1630 (2014), DOI: 10.1063/1.4902786

7. KM3NeT 2.0 Letter of Intent for ARCA and ORCA, arXiv:1601.07459 [astro-ph.IM]27 Jan 2016 